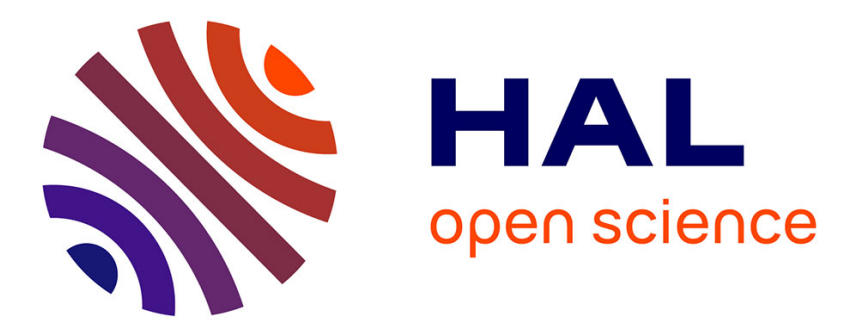

\title{
Low density shear viscosity of Lennard-Jones chains of variable rigidities
}

Stephanie Delage Santacreu, Guillaume Galliero, Marc Odunlami, Christian Boned

\section{- To cite this version:}

Stephanie Delage Santacreu, Guillaume Galliero, Marc Odunlami, Christian Boned. Low density shear viscosity of Lennard-Jones chains of variable rigidities. Journal of Chemical Physics, 2012, 137, pp.204306. 10.1063/1.4767528 . hal-00781993

\section{HAL Id: hal-00781993 https://hal.science/hal-00781993}

Submitted on 25 May 2021

HAL is a multi-disciplinary open access archive for the deposit and dissemination of scientific research documents, whether they are published or not. The documents may come from teaching and research institutions in France or abroad, or from public or private research centers.
L'archive ouverte pluridisciplinaire HAL, est destinée au dépôt et à la diffusion de documents scientifiques de niveau recherche, publiés ou non, émanant des établissements d'enseignement et de recherche français ou étrangers, des laboratoires publics ou privés. 


\title{
Low density shear viscosity of Lennard-Jones chains of variable rigidities
}

\author{
S. Delage Santacreu, ${ }^{1}$ G. Galliero, ${ }^{2}$ M. Odunlami, ${ }^{3}$ and C. Boned ${ }^{2}$ \\ ${ }^{1}$ Pôle Calcul Scientifique et Traitement des données, Université de Pau et des Pays de l'Adour, rue Jules Ferry, \\ 64000 PAU, France \\ ${ }^{2}$ Laboratoire des Fluides Complexes et leurs Reservoirs (UMR-5150 with CNRS and TOTAL), Université de \\ Pau et des Pays de l'Adour, BP 1155, F-64013 PAU Cedex, France \\ ${ }^{3}$ Laboratoire de Mathématiques Appliquées (UMR-5142 with CNRS), Université de Pau et des Pays de \\ l'Adour, BP 1155, F-64013 PAU Cedex, France
}

(Received 12 September 2012; accepted 31 October 2012; published online 27 November 2012)

\begin{abstract}
The zero-density shear viscosity of different types of short Lennard-Jones chains, up to the hexadecamer, has been evaluated using a non-equilibrium molecular dynamics scheme. Simulations have been performed on chains of variable rigidities going from the fully flexible to the fully rigid chains. Very interestingly, it is found that there exists a universal relation (a power law) between the zerodensity viscosity of the Lennard-Jones chains and their radius of gyration whatever the rigidity of the chain and for all tested temperatures (ranging from 2.5 to 6 in reduced units). Furthermore, for the studied range of temperature, it is shown that the zero-density viscosity of both fully flexible chains and fully rigid chains models can be obtained with an accuracy of a few percents knowing only the dimer viscosity and the length of the chain. () 2012 American Institute of Physics. [http://dx.doi.org/10.1063/1.4767528]
\end{abstract}

\section{INTRODUCTION}

Despite continuous progress, there is still no general approach for an exact estimation of shear viscosity applicable for all fluid states. ${ }^{1-8}$ This is largely due to a lack of a comprehensive theory for evaluating the transport properties in dense fluids in terms of a realistic molecular model, i.e., molecular structure and interaction potentials. This problem is even more pronounced when dealing with poly-atomic fluids, even if there are recent improvements on that topic. ${ }^{7-13}$ When dealing with low density situations, kinetic theories and all related approaches ${ }^{1,3,14}$ have proven to be accurate to predict viscosity of simple molecular models. Hence, they can be used with a reasonable success to describe the viscosity of simple real fluids, ${ }^{2}$ but, when polyatomic molecules are involved, the problem is by far more complex. In recent years, major theoretical advances have been made in particular in order to relate the viscosity to a single generalized cross section (see, for example, Court et al. ${ }^{15}$ ) together with progress on how to perform accurate calculations. ${ }^{16}$ However, parameters that quantify the efficiency with which internal and translational energies are exchanged during collisions are rarely known in most cases and so such approaches are not always simple to apply. It should be noticed that one way to circumvent this difficulty is to employ molecular dynamics (MD) simulations ${ }^{17}$ or the classical trajectory method, ${ }^{18}$ which allows a direct computation of the transport properties with a precision that can be of the order of the experimental one. ${ }^{16,19,20}$

One of the most popular poly-atomic molecular models to describe fluid molecules is the Lennard-Jones chain (LJC) model, which is composed of freely jointed spheres interacting through a Lennard-Jones potential. This fluid model, despite its simplicity, has shown to be valuable to describe thermodynamic properties of some polyatomic fluids, e.g., normal alkanes $^{21}$ as well as interfacial properties ${ }^{22}$ or even transport properties in some cases. ${ }^{8-10,12,23,24}$ So, as this fluid model is rather simple, one is tempted to develop a scheme based on this molecular model, or a similar one, aiming at predicting the viscosity of polyatomic fluids as in Refs. 9, 10, and 12. However, such a scheme needs an accurate estimate of the zero-density viscosity of these fluid models (i.e., the "translational/kinetic" viscosity, which is the shear viscosity of an infinitely dilute fluid) as a starting point, in particular those based on an Enskog-like approach. As long as such quantity is not accessible yet theoretically, one way to proceed is to use adequate molecular dynamics simulations that allow to obtain directly the zero-density viscosity for a given molecular model. ${ }^{10,25,26}$

Thus, to improve the modeling of the zero-density shear viscosity of LJC fluids and to extend the work initiated in Ref. 10 about the shear viscosity of fully flexible LJC, numerous non-equilibrium molecular dynamics (NEMD) simulations on short Lennard-Jones chain in low density states have been performed. In addition, as the internal degrees of freedom are important when dealing with transport properties, we have not only studied the fully flexible LJC fluid but as well LJC fluids of variable rigidities up to the fully rigid LJC fluid. Furthermore, it will be shown how the zero-density viscosity of this family of fluid models can be simply related to their radius of gyration. Finally, relations are proposed to accurately describe the zero-density viscosity of the fully flexible and the fully rigid LJC fluid models (up to the hexadecamer) knowing only the dimer viscosity and the length of the chain.

The manuscript is structured as follows. In Sec. II, we briefly describe the fluid model studied in this work and we provide numerical details as well as some basics on the viscosity modeling in dilute state. In Sec. III, we first indicate how is computed the zero-density viscosity using NEMD 
simulations. Then, we provide some results for fully flexible and fully rigid chains. These results are analyzed and modeled in terms of chain length for both type of chains. Then, it is shown how the zero-density of Lennard-Jones chains of variable rigidities (ranging from the fully flexible to the fully rigid) can be universally related to the radius of gyration. Finally, the conclusions of this study are drawn in Sec. IV.

\section{MODELS AND THEORY}

\section{A. The LJC fluid model}

As mentioned previously, we consider in this study Lennard-Jones chain fluid models. Each molecule is described as a chain (homo-nuclear) of $N_{c}$ tangent spheres with bond lengths constrained. Inter-molecular and non-bonded intra-molecular interactions of non-adjacent spheres are described by the truncated Lennard-Jones $12-6$ potential, ${ }^{17}$

$$
U_{\mathrm{LJ}}=\left\{\begin{array}{ll}
4 \varepsilon\left[\left(\frac{\sigma}{r}\right)^{12}-\left(\frac{\sigma}{r}\right)^{6}\right], & \text { if } r<r_{c} \\
0, & \text { if } r \geq r_{c}
\end{array},\right.
$$

where $\varepsilon$ is the potential strength, $\sigma$ the sphere "diameter", $r$ is the centre to centre distance between the two spheres considered, and $r_{c}$ is the cutoff radius (equal to $2.5 \sigma$ in this study).

Concerning bonded interactions, first, to maintain the bond length between adjacent spheres equal to $\sigma$, we have employed the classical RATTLE algorithm. ${ }^{27}$ Second, to include a "rigidity" parameter of the chain, we have introduced a simple harmonic bending potential described by ${ }^{28}$

$$
U_{\text {Bend }}=k\left(\theta-\theta_{0}\right)^{2},
$$

where $\theta_{0}$ is the equilibrium angle $\left(=180^{\circ}\right)$ between three adjacent spheres and $k$ is a stiffness constant. It should be noticed that when $k=0$ the molecule is fully flexible (i.e., it corresponds to the usual LJC model) and when $k \rightarrow \infty$ the molecule becomes fully rigid and behaves as a rod.

In the following, to simplify the analysis of the results, we use the classical LJ dimensionless units, namely,

$$
T^{*}=\frac{k_{B} T}{\varepsilon}, \quad \rho^{*}=\frac{N_{T} \sigma^{3}}{V}, P^{*}=\frac{P \sigma^{3}}{\varepsilon}, \eta^{*}=\eta \frac{\sigma^{2}}{\sqrt{m \varepsilon}}, k^{*}=\frac{k}{\varepsilon},
$$

where $k_{B}$ is the Boltzmann constant, $T$ is the temperature, $N_{T}$ is the total number of spheres in the simulation box (i.e., $N_{c}$ $\times N_{\text {mol }}$, where $N_{\text {mol }}$ is the number of molecules), $V$ is the volume of the simulation box, $P$ is the pressure, $m$ is the mass of the monomer, and $\eta$ is the shear viscosity of the chain. It is important to note that, using this set of reduced variables, the LJC fluid models used in this study are completely defined by their length, $N_{c}$, and their rigidity, $k$.

\section{B. Simulation context}

A in-house code already validated on various fluid types ${ }^{10,29,30}$ is used to perform the molecular dynamics simulations. The equations of motion are integrated by using the velocity Verlet algorithm ${ }^{17}$ together with the RATTLE approach. ${ }^{27}$ Classical periodic boundaries with Verlet neighbor's lists are employed and a Berendsen thermostat ${ }^{31}$ is ap- plied to maintain the desired temperature. Error bars have been computed using the sub-block method. ${ }^{17}$ Several chain lengths have been studied $\left(1 \leq N_{c} \leq 16\right)$ with a specific amount of molecules $N_{\text {mol }}$. For $N_{c}=1$ (respectively, 2, 3, 4, $6,8,10,12,16$ ), we use $N_{\text {mol }}=1500$ (respectively, 750, 500, $375,250,200,200,200,200)$.

To estimate the shear viscosity of the simulated fluids, the boundary driven nonequilibrium scheme of Müller-Plathe ${ }^{32}$ has been applied with a subdivision of the simulation box in 24 slabs. To generate the shear, an exchange frequency equal to 500 has been chosen to avoid any shear thinning, ${ }^{10,33}$ a point that has been numerically checked. The slabs where the exchanges are performed, as well as their first neighbors, have been discarded to measure the shear rate. Simulations have been performed with an equilibration phase of more than $10^{6}$ time steps, a transient state of more than $10^{6}$ nonequilibrium time steps and a steady state of more than $1.5 \times 10^{7}$ nonequilibrium time steps. The viscosity is computed and averaged during the steady state of the nonequilibrium phase. Using these parameters, the error bars have been found to be lower than $5 \%$ in most cases. In the following, only results corresponding to stable states have been provided.

\section{Zero-density viscosity modeling}

Away from the critical point, the shear viscosity is usually decomposed as s, $^{3,2}$

$$
\eta=\eta_{0}+\eta_{r}
$$

where $\eta_{0}$ is the zero-density contribution to the viscosity and $\eta_{r}$ is the residual viscosity. The zero-density viscosity represents the contribution to the viscosity that is independent of density (but not of temperature) and corresponds to the viscosity of a fluid in a very dilute state, i.e., when $\rho \rightarrow 0$. For a Lennard-Jones (monomer) fluid, the zero-density viscosity can be accurately evaluated ${ }^{15}$ by using the Chapman-Enskog solution to the Boltzmann equation, namely,

$$
\eta_{0, \mathrm{LJ}}^{*}=\frac{5}{16 \Omega_{v}} \sqrt{\frac{T^{*}}{\pi}}
$$

where $\Omega_{v}$ is the collision integral that can be estimated using the accurate correlation provided by Neufeld et al. ${ }^{34}$

Unfortunately for chain-like molecules, the problem is more complex despite progress made in order to relate viscosity to a single generalized cross section that is proportional to the more traditional collision integral. ${ }^{15}$ Among the general trends, one can expect a decrease of the zero-density viscosity when the chain length increases because of the conversion of translation into rotation in a chain like molecule. This assumption is supported by experimental results on chain-like fluids such as normal alkanes, for which a decrease of the low density viscosity when the chain length increases is noted for a given temperature. ${ }^{35}$ From the modeling point of view, some relations, based on different assumptions ${ }^{10,12}$ have been proposed recently to describe the zero-density viscosity of a fully flexible chain-like fluid, $\eta_{0 \text {,chain, }}$ starting from the knowledge of the value of the monomer, $\eta_{0, \text { mono: }}$ : 
(1) the proposal of Galliero and Boned,${ }^{10}$ developed for the fully flexible LJC fluid,

$$
\eta_{0, \text { chain }}^{*}=\frac{\eta_{0, \text { mono }}^{*}}{N_{c}^{1 / 2}},
$$

(2) and the model of Zhang and $\mathrm{Yu},{ }^{12}$ constructed to deal with freely jointed hard-spheres in dense states and extrapolated to the zero-density limit,

$$
\eta_{0, \text { chain }}^{*}=\frac{F_{\eta}\left(N_{c}, \rho^{*} \rightarrow 0\right) \Delta \gamma \eta_{0, \text { mono }}^{*}}{N_{c}^{1 / 6} \exp \left(-0.4 / T^{* 1.5}\right)},
$$

with $\gamma=1, \Delta=1.218$, and $F_{\eta}$ a function defined in Ref. 12.

\section{RESULTS}

\section{A. MD computation of the zero-density viscosity}

Molecular dynamics is not well suited to deal with very low density systems, even though it has been used under such conditions. ${ }^{26}$ So, the idea is to compute the shear viscosity at a small but non-null density and to deduce the zero-density viscosity from this computation. For that purpose, we have used an approach similar to the one proposed in Ref. 10 with some modifications. In that approach, we take advantage of the fact that the linear momentum flux (the shear stress), $J_{x z}$, is directly provided by the NEMD scheme $\mathrm{e}^{10,32}$ and can be computed as well using its microscopic formulation which is for an atomic fluid:

$$
J_{x z}^{*}=-\frac{1}{V^{*}}\left(\sum_{i=1}^{N_{T}} v_{i, x}^{*} v_{i, z}^{*}+\sum_{i=1}^{N_{T}-1} \sum_{j>i}^{N_{T}} r_{i j, x}^{*} F_{i j, z}^{*}\right),
$$

where $N_{T}$ is the number of spheres, $V^{*}$ is the dimensionless domain volume, $v_{i, x}^{*}$ is the $x$ component of the velocity of particle $i, v_{i, z}^{*}$ is the $z$ component of the velocity of particle $i, r_{i j, x}^{*}$ is the $x$ component of the distance between particles $i$ and $j$, and $F_{i j, z}^{*}$ is the $z$ component of the force of particle $i$ acting on particle $j$.

The first term of Eq. (8) represents the translational contribution, while the second one represents the collisional contribution. It is also possible to define straightforwardly and to compute during MD simulations ${ }^{10,25}$ a translational/kinetic viscosity, $\eta_{t}$, and a collisional/configurational viscosity, $\eta_{c}$, associated, respectively, with the two contributions of the shear stresses appearing in Eq. (8). By definition, $\eta_{c}$ tends towards zero when the density decreases. Hence, we can deduce that

$$
\lim _{\rho \rightarrow 0} \eta_{t}=\eta_{0}
$$

Strictly speaking, $\eta_{t}=\eta_{0}$ when $\rho=0$; however, one may be tempted to quantify the limits of this equality when the density is not equal to zero, as long as MD simulations cannot be performed at $\rho=0$. To study that point, we have computed the translational viscosity of the $\mathrm{LJ}\left(N_{c}=1\right)$ fluid at a supercritical temperature $T^{*}=3$ for different densities varying from $\rho^{*}=0.05$ to $\rho^{*}=0.8$.

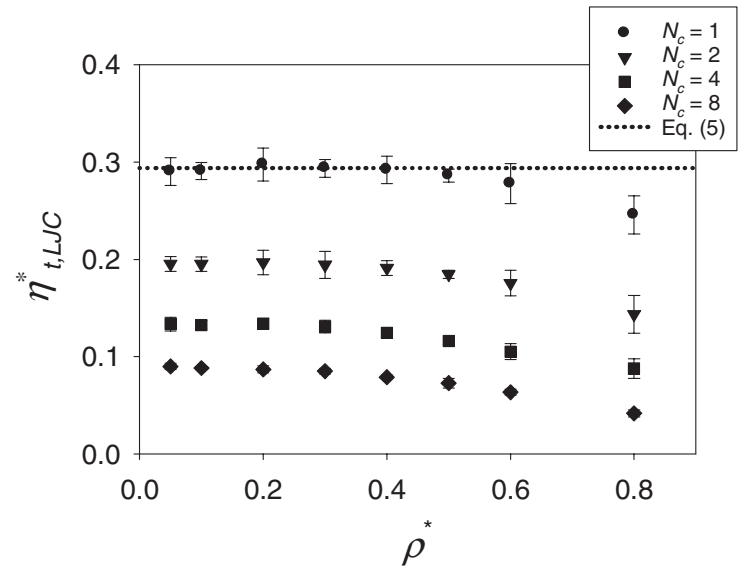

FIG. 1. Translational viscosity of the fully flexible LJC fluid versus density for different chain lengths $\left(N_{c}=1,2,4\right.$, and 8$)$ and for $T^{*}=3$. The dotted line corresponds to the zero-density viscosity of the monomer.

As can be seen in Figure 1, the translational viscosity is a very good approximation of the zero-density viscosity when the density is sufficiently low. However, when the density increases the translational viscosity decreases. This can be understood because when density increases the free volume accessible to displacement decreases (exchange of momentum by translation), and this decrease is more pronounced than the increase of the density (number of particles transported) and so (see Eq. (8)) the translational viscosity should decrease when density increases.

Interestingly, when $\rho^{*} \leq 0.4$, the deviation between $\eta_{t, \mathrm{LJ}}$ and $\eta_{0, \mathrm{LJ}}$ computed using Eq. (4) is smaller than $1.5 \%$ in all cases. To verify the applicability of such a scheme to estimate the zero-density of the LJC fluid (for $N_{c}>1$ ), one has first to compute its translation viscosity. For molecular fluids, a formulation using the coordinates and velocities of the barycentre of molecules should be used. We note $\bar{x}_{j}=\frac{1}{N_{c}} \sum_{i=1}^{N_{c}} x_{j, i}$ (resp., $\bar{z}_{j}$ ) the $x$ component of the coordinates of a molecule $j$, where $x_{j, i}, i \in \llbracket 1, N_{c} \rrbracket$ are the $x$ components of the coordinates of particles $i$ belonging to a molecule $j$. In the same way, the $x$ component of the velocity of a molecule $j$ is defined as $\bar{v}_{j, x}^{*}=\frac{1}{N_{c}} \sum_{i=1}^{N_{c}} v_{j, x, i}^{*}$. As a result, the linear momentum flux associated with the shear stress of LJC fluids (whatever the rigidity of the chain) can be formulated as follows:

$$
\begin{aligned}
J_{x z, t}^{* \mathrm{LJC}} & =\frac{-1}{V^{*}} \sum_{j=1}^{N_{\mathrm{mol}}} \bar{v}_{j, x}^{*} \bar{v}_{j, z}^{*} \\
& =\frac{-1}{V^{*} N_{c}^{2}} \sum_{j=1}^{N_{\mathrm{mol}}}\left[\left(\sum_{i=1}^{N_{c}} v_{j, x, i}^{*}\right)\left(\sum_{i=1}^{N_{c}} v_{j, z, i}^{*}\right)\right] .
\end{aligned}
$$

Using that formulation, similarly to what done for the LJ fluid $\left(N_{c}=1\right)$, we have computed the translational viscosities of the fully flexible LJC fluid model $(k=0)$ for several chain lengths $\left(N_{c}=2,4\right.$, and 8$)$ and different densities, from $\rho^{*}=0.05$ to $\rho^{*}=0.8$, at $T^{*}=3$.

The translational viscosity of the fully flexible LJC fluids increases when density decreases as clearly exhibited in Figure 1 . Then, similarly to the LJ fluid, $\eta_{t, \mathrm{LJC}}^{*}$ reaches a plateau for all chain lengths. It seems that this plateau tends 


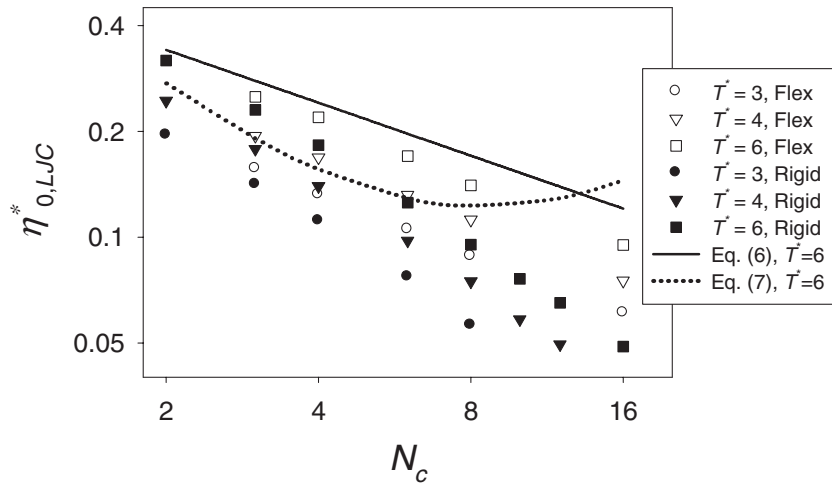

FIG. 2. Zero-density viscosity of the fully flexible and rigid LJC models for different chain lengths and different temperatures. Error bars on MD results are smaller than symbol size.

to start for smaller values of $\rho^{*}$ when $N_{c}$ increases. However when $\rho^{*} \leq 0.2$, the deviations between the values obtained are of the order of the error bar. Thus, in the following, to estimate the zero-density viscosity of the LJC fluids we have assumed that

$$
\eta_{0, \mathrm{LJC}}^{*}=\eta_{t, \mathrm{LJC}}^{*}\left(\rho^{*}=0.1\right)
$$

\section{B. Modeling of the zero-density viscosity of Lennard-Jones chains}

The aim of this subsection is to correlate the zero-density viscosity with the chain length for fully flexible and fully rigid Lennard-Jones chains for the studied range of temperatures. For that purpose, using the scheme described in Sec. III A, the zero-density viscosity has been computed using different chain lengths $\left(2 \leq N_{c} \leq 16\right)$, various temperatures (2.5 $\leq T^{*} \leq 6$ ), and two spring constants $k^{*}=0$ (fully flexible molecules) and $k^{*}=1000$ (rigid molecules), for a total of 45 different systems. By computing the end to end vector ${ }^{35}$ during the simulations, it has been verified that using $k^{*}$ $=1000$ corresponds to a practically fully rigid linear molecule, the end to end distance being equal to $N_{c} \times \sigma$ with deviations below $1.5 \%$. The obtained zero-density viscosity data can be found in Table I.

As shown on Figure 2, for both flexible and rigid chains, the zero-density viscosity decreases monotonically when $N_{c}$ increases. In addition, the decrease of $\eta_{0, \mathrm{LJC}}^{*}$ with $N_{c}$ is noticeably more pronounced when dealing with fully rigid chains compared with fully flexible chains. This behavior can be understood as, for a given chain length, rigid chain "occupies" more space (see Sec. III C) and so should be less viscous than the corresponding flexible chain. Interestingly, it seems that, for both LJC types, the dependence of $\eta_{0, \mathrm{LJC}}^{*}$ with $N_{c}$ is of a power-law type with an exponent independent of temperature. Furthermore, as for spherical molecular models, ${ }^{14} \eta_{0, \text { LJC }}^{*}$ increases when the temperature increases.

Concerning the modeling of these results, it appears clearly in Figure 2 that Eq. (6), which was designed to deal with fully flexible chains, ${ }^{10}$ tends to overestimate $\eta_{0, \mathrm{LJC}}^{*}$ in all cases, whereas Eq. (7) leads to an underestimation for short
TABLE I. Translational viscosity and radius of gyration of LJC fluids at $\rho^{*}$ $=0.1$, for different chain lengths $\left(2 \leq N_{c} \leq 16\right)$, temperatures $\left(3 \leq T^{*} \leq 6\right)$, and different stiffnesses $\left(0 \leq k^{*} \leq 1000\right)$.

\begin{tabular}{|c|c|c|c|c|}
\hline$k^{*}$ & $N_{c}$ & $T^{*}$ & $\eta_{0, \mathrm{LJC}}^{*}$ & $R_{g}^{*}$ \\
\hline 0 & 2 & 3 & $0.196 \pm 0.005$ & 1.000 \\
\hline 0 & 3 & 3 & $0.157 \pm 0.002$ & 1.192 \\
\hline 0 & 4 & 3 & $0.132 \pm 0.003$ & 1.349 \\
\hline 0 & 6 & 3 & $0.105 \pm 0.001$ & 1.619 \\
\hline 0 & 8 & 3 & $0.088 \pm 0.001$ & 1.825 \\
\hline 0 & 2 & 4 & $0.244 \pm 0.005$ & 1.000 \\
\hline 0 & 3 & 4 & $0.194 \pm 0.003$ & 1.196 \\
\hline 0 & 4 & 4 & $0.169 \pm 0.001$ & 1.358 \\
\hline 0 & 6 & 4 & $0.132 \pm 0.003$ & 1.622 \\
\hline 0 & 8 & 4 & $0.112 \pm 0.003$ & 1.853 \\
\hline 0 & 16 & 4 & $0.075 \pm 0.001$ & 2.578 \\
\hline 0 & 2 & 6 & $0.318 \pm 0.007$ & 1.000 \\
\hline 0 & 4 & 6 & $0.219 \pm 0.006$ & 1.359 \\
\hline 0 & 6 & 6 & $0.170 \pm 0.005$ & 1.627 \\
\hline 0 & 8 & 6 & $0.141 \pm 0.002$ & 1.856 \\
\hline 0 & 16 & 6 & $0.095 \pm 0.004$ & 2.631 \\
\hline 10 & 3 & 3 & $0.152 \pm 0.002$ & 1.280 \\
\hline 10 & 4 & 3 & $0.124 \pm 0.001$ & 1.530 \\
\hline 10 & 6 & 3 & $0.087 \pm 0.002$ & 1.979 \\
\hline 10 & 8 & 3 & $0.068 \pm 0.001$ & 2.381 \\
\hline 10 & 12 & 3 & $0.046 \pm 0.003$ & 3.088 \\
\hline 10 & 4 & 6 & $0.201 \pm 0.003$ & 1.476 \\
\hline 10 & 8 & 6 & $0.121 \pm 0.002$ & 2.185 \\
\hline 10 & 16 & 6 & $0.073 \pm 0.002$ & 3.223 \\
\hline 100 & 4 & 3 & $0.118 \pm 0.001$ & 1.608 \\
\hline 100 & 4 & 6 & $0.192 \pm 0.002$ & 1.599 \\
\hline 100 & 8 & 6 & $0.101 \pm 0.002$ & 2.695 \\
\hline 1000 & 3 & 3 & $0.141 \pm 0.004$ & 1.316 \\
\hline 1000 & 4 & 3 & $0.111 \pm 0.003$ & 1.617 \\
\hline 1000 & 6 & 3 & $0.077 \pm 0.002$ & 2.205 \\
\hline 1000 & 8 & 3 & $0.056 \pm 0.002$ & 2.786 \\
\hline 1000 & 3 & 4 & $0.178 \pm 0.004$ & 1.312 \\
\hline 1000 & 4 & 4 & $0.140 \pm 0.001$ & 1.617 \\
\hline 1000 & 6 & 4 & $0.098 \pm 0.002$ & 2.204 \\
\hline 1000 & 8 & 4 & $0.075 \pm 0.001$ & 2.785 \\
\hline 1000 & 10 & 4 & $0.058 \pm 0.002$ & 3.362 \\
\hline 1000 & 12 & 4 & $0.050 \pm 0.003$ & 3.936 \\
\hline 1000 & 3 & 6 & $0.230 \pm 0.005$ & 1.316 \\
\hline 1000 & 4 & 6 & $0.183 \pm 0.002$ & 1.616 \\
\hline 1000 & 6 & 6 & $0.126 \pm 0.002$ & 2.202 \\
\hline 1000 & 8 & 6 & $0.095 \pm 0.001$ & 2.781 \\
\hline 1000 & 10 & 6 & $0.076 \pm 0.003$ & 3.356 \\
\hline 1000 & 12 & 6 & $0.065 \pm 0.002$ & 3.928 \\
\hline 1000 & 16 & 6 & $0.049 \pm 0.001$ & 5.066 \\
\hline
\end{tabular}

chains and overestimation for the longer chains. Concerning the trend obtained with Eq. (7), this is not surprising as long as this model ${ }^{12}$ has not being designed specifically for the fully flexible, neither the fully rigid, Lennard-Jones chains. This indicates that these models, Eqs. (6) and (7), may lead to difficulties when being applied to predict the shear viscosity of real fluids adequately described by LJC at least in low density conditions.

To define an alternative to Eqs. (6) and (7) for both types of chains, one has to define a reference fluid model valid for the two types of chains. As shown in Figure 2, the 


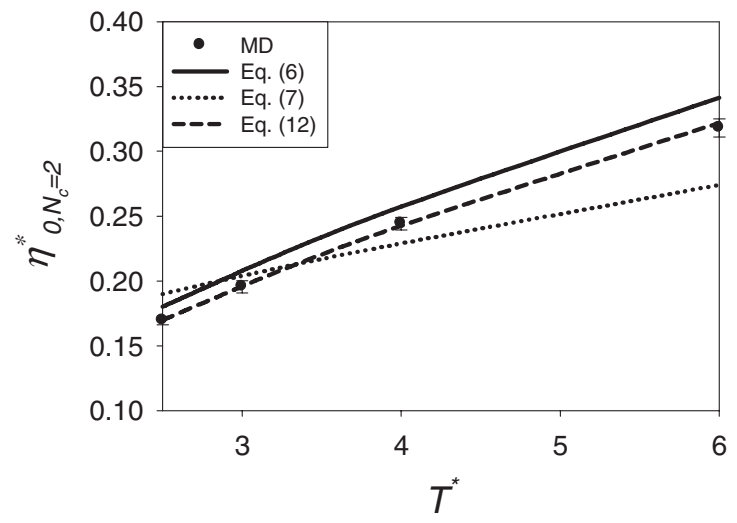

FIG. 3. Zero-density viscosity of the LJC dimer for different temperatures.

alternative relation should be dependent on the rigidity when $N_{c} \geq 2$ but should be independent of the rigidity when going from the monomer to the dimer, as long as there is no difference between the "flexible" dimer and the "rigid" dimer. Thus, it makes sense to choose the dimer, $N_{c}=2$, as the reference fluid and not the monomer. To describe the zero-density viscosity of the LJC dimer, we have first tested Eqs. (6) and (7) combined with Eq. (4) against the data provided by the MD simulation. As shown in Figure 3, Eq. (6) provides reasonable results (with a slight overestimation) for the dimer, whereas Eq. (7) does not provide the correct temperature dependence. As a simple and accurate alternative for the zerodensity viscosity of the LJC dimer, we propose the following relation:

$$
\eta_{0, N_{c}=2}^{*}=\frac{2}{3} \eta_{0, \mathrm{LJ}}^{*}=\frac{5}{24 \Omega_{v}} \sqrt{\frac{T^{*}}{\pi}}
$$

This relation, compared with the MD results for the studied range of temperatures, yields an average absolute deviation (AAD) equal to $0.6 \%$ with a maximum absolute deviation $(\mathrm{MxD})$ below $1.2 \%$.

\section{Fully flexible chains}

Using the data (see Figure 2), it appears that a powerlaw independent of temperature should be able to describe well the zero-density viscosity of the fully flexible LJC using the dimer as a reference. Thus, $\eta_{0, \mathrm{LJC}}^{*}$ should be proportional to $\left(\frac{N_{c}}{2}\right)^{\alpha}$, where $\alpha$ is a numerical parameter adjusted on the MD data. For the fully flexible chain (for $N_{c} \geq 2$ ), we have obtained

$$
\eta_{0, \mathrm{LJC} \text { flex }}^{*}=\frac{\eta_{0, N_{c}=2}^{*}}{\left(\frac{N_{c}}{2}\right)^{0.57}}=\frac{5}{24 \Omega_{v}\left(\frac{N_{c}}{2}\right)^{0.57}} \sqrt{\frac{T^{*}}{\pi}}
$$

The obtained value $(\alpha=-0.57)$ will be discussed in Sec. III C.

When Eq. (13) is employed to describe the zero-density viscosity of the fully flexible LJC fluid (for $2 \leq N_{c} \leq 16$ and $2.5 \leq T^{*} \leq 6$ ), it yields, compared with MD results (24 points), an $\mathrm{AAD}=1.5 \%$ with a $\mathrm{MxD}=4.3 \%$. This result can be considered as excellent taken into account the uncertainties of the NEMD data.

It should be noticed that, by combining Eqs. (12) and (13), it is possible to obtain another relation for the fully flexible LJC, i.e., $\eta_{0, \mathrm{LJC} \text { flex }}^{*}=\frac{5}{16 \Omega_{v} N_{c}^{0.57}} \sqrt{\frac{T^{*}}{\pi}}$ because $2^{-0.57} \approx 2 / 3$.

\section{Fully rigid chains}

Similarly to what was done for the fully flexible chain, it can be seen from Figure 2 that a relation of the type of Eq. (13) should be suitable to describe the zero-density viscosity of a rigid Lennard-Jones chain but with a different exponent $\alpha$. By fitting the MD data on the fully rigid chain, we get $\alpha=-0.88$, a value that will be discussed in Section III C and so for $N_{c} \geq 2$ :

$$
\eta_{0, \mathrm{LJC} \text { rigid }}^{*}=\frac{\eta_{0, N_{c}=2}^{*}}{\left(\frac{N_{c}}{2}\right)^{0.88}}=\frac{5}{24 \Omega_{v}\left(\frac{N_{c}}{2}\right)^{0.88}} \sqrt{\frac{T^{*}}{\pi}} .
$$

When this model is employed to describe the MD zerodensity viscosity of the fully rigid LJC fluid (for $2 \leq N_{c}$ $\leq 16$ and $3 \leq T^{*} \leq 6$ ), it provides an $\mathrm{AAD}=2.8 \%$ with a $\mathrm{MxD}=5.7 \%$ (20 points) which is good but slightly poorer than what obtained for a fully flexible chain.

\section{Universal relation between the zero-density viscosity of chains of variable rigidities and the radius of gyration}

From the results obtained previously, it appears that the zero-density viscosity of a chain divided by its dimer value can be simply related to the chain length. This probably indicates that the zero-density viscosity of a chain, flexible or rigid, can be related to a geometric quantity describing its effective size. In the following, we will show that this statement is valid and that the radius of gyration is the appropriate geometric quantity to describe the effective size of the chains studied in this study.

The average radius of gyration, $R_{g}$, of $N_{\text {mol }}$ long chains $i$ composed of $N_{c}$ particles $j$ reduced to points located at $M_{i, j}$ and linked by segments of dimension $\sigma$, is given by ${ }^{36}$

$$
\left|\boldsymbol{R}_{\boldsymbol{g}}\right|^{2}=\frac{1}{N_{\mathrm{mol}}} \sum_{i=1}^{N_{\text {mol }}} \frac{1}{N_{c}} \sum_{j=1}^{N_{c}}\left|\boldsymbol{G}_{\boldsymbol{i}} \boldsymbol{M}_{\boldsymbol{i}, \boldsymbol{j}}\right|^{2},
$$

where $G_{i}$ is the barycenter of the chain $i$. However, because we are dealing here with short chains, we have to take into account the size of the spheres and so,

$$
\left|\boldsymbol{R}_{\boldsymbol{g}}\right|=\sqrt{\frac{1}{N_{c} N_{\mathrm{mol}}} \sum_{i=1}^{N_{\mathrm{mol}}} \sum_{j=1}^{N_{c}}\left|\boldsymbol{G}_{\boldsymbol{i}} \boldsymbol{M}_{\boldsymbol{i}, \boldsymbol{j}}\right|^{2}}+\frac{\sigma}{2} .
$$

Thus, for different temperatures ranging from $T^{*}=3$ to $T^{*}=6$, different chain lengths with $N_{c}$ ranging from 2 to 16 and different rigidities $k^{*}=0,10,100$, and 1000, the radius of gyration, and the zero-density viscosity have been computed. Values are provided in Table I.

As expected, the radius of gyration is increasing when $N_{c}$ increases and when $k$ increases (see Figure 4 and Table I). 


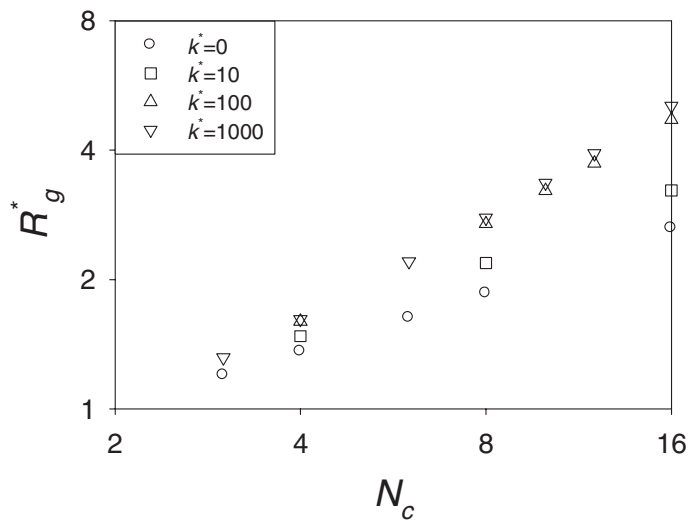

FIG. 4. Radius of gyration versus chain length for different rigidities at $T^{*}=6$.

Furthermore, the relation between the radius of gyration and the chain length seems to be of the power law form. We will address that point in the following. Very interestingly, as shown in Figure 5, the ratio $\eta_{0, \mathrm{LJC}}^{*} / \eta_{0, N_{c}=2}^{*}$ seems to be a unique function of the radius of gyration whatever the temperature, the length, and the rigidity of the chain. In addition, we have noticed that a simple power law of the form

$$
\frac{\eta_{0, \mathrm{LJC}}^{*}}{\eta_{0, N_{c}=2}^{*}}=\left(R_{g}^{*}\right)^{\beta}
$$

with $\beta=-1.2$ is able to consistently represent the full set of data obtained as shown in Figure 5 for the studied range of temperatures. Such a result indicates that, for the LJ short chains studied here, flexible or not, the knowledge of the radius of gyration provides directly a very good estimate of the zero-density viscosity if the dimer value is known. More precisely, when combining Eqs. (12) and (17), one obtains, when $N_{c} \geq 2$,

$$
\eta_{0, \mathrm{LJC}}^{*}=\frac{5}{24 \Omega_{v}\left(R_{g}^{*}\right)^{1.2}} \sqrt{\frac{T^{*}}{\pi}},
$$

which leads to an $\mathrm{AAD}=3.3 \%$ with a MxD below 9\% compared to the MD results of all types of LJ chain, from fully flexible to fully rigid.

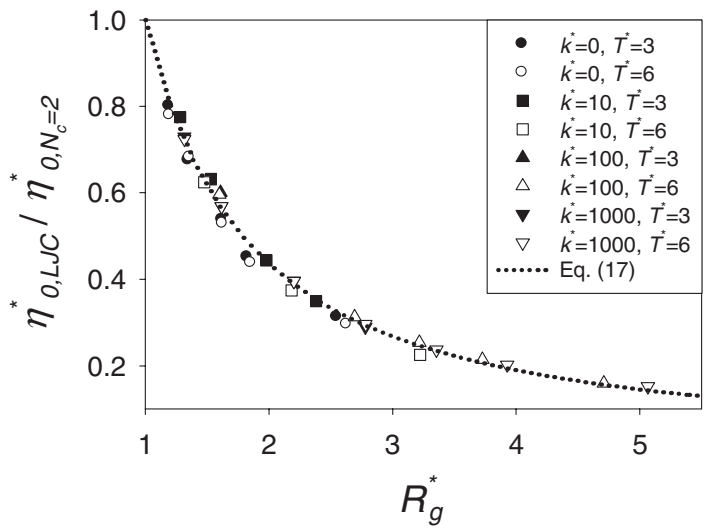

FIG. 5. Variations of the zero-density viscosity versus the radius of gyration for different temperatures, chain lengths, and rigidities.
The universal law so obtained, Eq. (18), provides an explanation of the values of $\alpha$ obtained when relating $\eta_{0, \mathrm{LJC}}^{*}$ to the chain length, Eqs. (13) and (14). It is well known ${ }^{37}$ that a freely jointed chain (ideal chain) yields a radius of gyration proportional to $\left(N_{c} / 2\right)^{0.5}$. Thus, a freely jointed LJC should lead to a similar result, even if there exist several differences between the two chain models. In fact, when combining Eqs. (13) and (18), one obtains a radius of gyration of the fully flexible LJC proportional to $N_{c}{ }^{0.475}$ which is consistent with what known for an ideal chain. Concerning the fully rigid linear chain, the reduced radius of gyration can be estimated analytically ${ }^{37}$ and is equal to $\sqrt{\frac{\left(N_{c}-1\right)\left(N_{c}+1\right)}{12}}+\frac{1}{2}$. In this study, when combining Eqs. (14) and (18), we can deduce that $R_{g}$ is equal to $\left(N_{c} / 2\right)^{0.733}$ for the fully rigid LJC. Despite not being exact, this relation is a good approximation of the analytical solution, the $\mathrm{MxD}$ between the two relations being $10 \%$ when $N_{c} \leq 16$.

\section{CONCLUSION}

To estimate the zero-density shear viscosity of short Lennard-Jones chains, a nonequilibrium molecular dynamics scheme is proposed in this article. The simulations have been performed for various supercritical temperatures, $T^{*}$ varying from 2.5 to 6 , and for various chain lengths from the dimer to the hexa-decamer. Furthermore, we have not only studied the fully flexible LJC but as well LJC of variable rigidities up to the fully rigid LJC.

The most striking result is that the zero-density viscosity of LJC is a unique function of the radius of gyration whatever the rigidity of the chain for the studied states, i.e., there exists a scaling law between the zero-density viscosity of a short chain and its radius of gyration. More precisely, a simple power law provides an estimation of the zero-density viscosity of any LJC (with $2 \leq N_{c} \leq 16$ ) knowing the radius of gyration with deviations below $9 \%$ for all cases tested here.

In addition, as the radius of gyration can be expressed as a simple power law function of the chain length for both the fully flexible and the rigid cases, two relations are proposed to accurately describe, respectively, the zero-density viscosity of the fully flexible LJC and the fully rigid LJC fluid models (up to the hexa-decamer) knowing only the dimer viscosity and the length of the chain. It is shown that these relations allow to obtain the zero-density viscosity of the two LJC models with deviations of the order of a few percent. We hope that this preliminary work and the proposed relations, which have only been tested for a relatively limited range of temperatures, could be useful in order to improve molecular based models aiming at predicting shear viscosities using a chain fluid model.

\section{ACKNOWLEDGMENTS}

We acknowledge the PCSTD (Pôle de Calcul Scientifique et de Traitement des Données) of the Université de Pau et des Pays de l'Adour (UPPA) and the MCIA (Mesocentre de Calcul Intensif Aquitain) of the Université de Bordeaux and of the UPPA for the computing facilities they provided us. 
${ }^{1}$ M. J. Assael, J. P. M. Trusler, and T. F. Tsolakis, Thermophysical Properties of Fluids (Imperial College Press, London, 1996).

${ }^{2}$ B. E. Poling, J. M. Prausnitz, and J. P. O'Connel, The Properties of Gases and Liquids (McGraw-Hill, New York, 2001).

${ }^{3}$ J. Millat, J. H. Dymond, and C. A. Nieto de Castro, Transport Properties of Fluids: Their Correlation, Prediction and Estimation (Cambridge University Press, Cambridge, 2005).

${ }^{4}$ D. S. Viswanath, T. K. Ghosh, D. H. L. Prasad, N. V. K. Dutt, and K. Y. Rani, Viscosity of Liquids (Springer, Dordrecht 2006).

${ }^{5}$ B. C. Eu, Transport Coefficients of Fluids, Springer Series in Chemical Physics, Vol. 82 (Springer, 2006).

${ }^{6}$ S. E. Quinones-Cisneros and U. K. Deiters, J. Phys. Chem. B 110, 12820 (2006).

${ }^{7}$ A. S. De Wijn, V. Vesovic, G. Jackson, and J. P. Martin Trusler, J. Chem. Phys. 128, 204901 (2008).

${ }^{8}$ G. Galliero, C. Boned, and J. Fernandez, J. Chem. Phys. 134, 064505 (2010).

${ }^{9}$ O. Suarez-Iglesias, I. Medina, C. Pizarro, and J. L. Bueno, Chem. Eng. Sci. 62, 6499 (2007).

${ }^{10}$ G. Galliero, and C. Boned, Phys. Rev. E 79, 021201 (2009).

${ }^{11}$ G. Galliero, and C. Boned, Phys. Rev. E 80, 061202 (2009).

${ }^{12}$ X. G. Zhang and Y. X. Yu, Fluid Phase Equilib. 295, 237 (2010).

${ }^{13}$ A. S. De Wijn, N. Riesco, G. Jackson, J. P. Martin Trusler, and V. Vesovic, J. Chem. Phys. 136, 074514 (2012)

${ }^{14} \mathrm{~S}$. Chapman and T. Cowling, The Mathematical Theory of Non-Uniform Gases (Cambridge University Press, Cambridge, 1981).

${ }^{15}$ F. R. W. McCourt, J. J. M. Beenakker, W. E. Köhler, and I. Kuscer, Nonequilibrium Phenomena in Polyatomic Gases (Oxford University Press, Oxford, 1990), Vols. 1 and 2.

${ }^{16}$ R. Hellmann, E. Bich, E. Vogel, and V. Vesovic, J. Chem. Eng. Data 57, 1312-1317 (2012).

${ }^{17}$ M. P. Allen and D. J. Tildesley, Computer Simulation of Liquids (Oxford University Press, USA, 1989).
${ }^{18}$ V. Vesovic and W. A. Wakeham, Int. Rev. Phys. Chem. 11, 161 (1992).

${ }^{19}$ Z. Liang and H. L. Tsai, Fluid Phase Equilib. 293, 196 (2010).

${ }^{20}$ R. Hellman, E. Bich, E. Vogel, and V. Vesovic, Phys. Chem. Chem. Phys. 13, 13749 (2011).

${ }^{21}$ F. Llovell, J. C. Pàmies, and L. F. Vega, J. Chem. Phys. 121, 10715 (2004).

${ }^{22}$ G. Galliero, J. Chem. Phys. 133, 074705 (2010).

${ }^{23}$ R. A. Reis, R. Nobrega, J. V. Oliveira, and F. W. Tavares, Chem. Eng. Sci. 60, 4581 (2005)

${ }^{24}$ M. Touzet, G. Galliero, V. Lazzeri, M. Z. Saghir, F. Montel, and J. C. Legros, C. R. Mec. 339, 318 (2011).

${ }^{25}$ S. Hess, Int. J. Thermophys. 23, 905 (2002).

${ }^{26}$ K. Meier, A. Laesecke, and S. Kabelac, J. Chem. Phys. 121, 3671 (2004).

${ }^{27}$ H. C. Andersen, J. Comput. Phys. 52, 24 (1983).

${ }^{28}$ M. Griebel, S. Knapek, and G. Zumbusch, Numerical Simulation in Molecular Dynamics, Texts in Computational Science and Engineering (Springer, Berlin, 2007).

${ }^{29}$ G. Galliero, C. Boned, and A. Baylaucq, Ind. Eng. Chem. Res. 44, 6963 (2005).

${ }^{30}$ G. Galliero, C. Nieto-Draghi, C. Boned, J. B. Avalos, A. D. Mackie, A. Baylaucq, and F. Montel, Ind. Eng. Chem. Res. 46, 5238 (2007).

${ }^{31}$ H. J. C. Berendsen, J. P. M. Postma, W. F. van Gunsteren, A. di Nola, and J. R. Haak, J. Chem. Phys. 81, 3684, (1984).

${ }^{32}$ F. Müller-Plathe, Phys. Rev. E 59, 48941999.

${ }^{33}$ M. Kröger, Phys. Rep. 390, 453 (2004).

${ }^{34}$ P. D. Neufeld, A. R. Janzen, and R. A. Aziz, J. Chem. Phys. 57, 1100 (1972).

${ }^{35}$ E. W. Lemmon, M. L. Huber, and M. O. McLinden, Reference Fluid Thermodynamic and Transport Properties, NIST Standard Reference Database 23, Version 8.0 (2007).

${ }^{36}$ R. J. Young and P. A. Lovell, Introduction to Polymers (CRC, New York, 2011).

${ }^{37}$ M. Doi and S. F. Edwards, The Theory of Polymer Dynamic (Oxford University Press, New York, 1998). 
The Journal of Chemical Physics is copyrighted by the American Institute of Physics (AIP). Redistribution of journal material is subject to the AIP online journal license and/or AIP copyright. For more information, see http://ojps.aip.org/jcpo/jcper/jsp 